to train and equip on-scene responders, and to coordinate responses to take advantage of the complementary skill sets of on-scene and EMS responders.

\section{EMS RESPONSE TIME TO SPORTS INJURIES: A ROLE FOR ON-SCENE RESPONDERS}

Viktor Bovbjerg, Sam Johnson, Marc Norcross. Oregon State University

\subsection{6/injuryprev-2020-savir.118}

Statement of Purpose Emergency medical services (EMS) often respond to injuries sustained during team athletics and sports. The interval between injury and EMS arrival allows on-scene responders to provide immediate care.

Methods/Approach We identified EMS runs associated with team or group sports (ICD-10 activity code Y93.6x), to a school or an athletic field or facility, using National Emergency Medical Services Information System (NEMSIS) 2017 data. We calculated EMS response times to scene as the difference between dispatch time and on-scene time. Initial patient acuity was defined as 'green' (lower acuity), 'yellow' (emergent), 'red' (critical), or 'black' (deceased). We compared mean response time by patient acuity group.

Results Of 1107 EMS runs identified, initial acuity for the majority was green $(764,70.2 \%)$, with a substantial yellow group (302, 27.8\%), and relatively few red cases (22, 2.0\%); there were no on-scene fatalities. Response times did not differ significantly by acuity. Approximately one quarter (292, $27.0 \%$ ) of EMS responses were within five minutes, with greater proportions in the 5-10 minute $(472,43.6 \%)$ and 10 + minute $(319,29.5 \%)$ range; all but 76 responses $(7 \%)$ were greater than two minutes.

Conclusions In over 90\% of EMS responses identified in the 2017 NEMSIS data involving response to a team sporting or athletic event, response time was greater than 2 minutes; approximately $75 \%$ were greater than 5 minutes. For every injury, but particularly for emergent and critical injuries, this represents an important interval during which patient assessment, stabilization, treatment, and packaging by on-scene responders could improve outcomes. Emergency action plans can formalize pre-EMS on-scene emergency response in athletic settings.

Significance and Contributions to Injury and Violence Prevention Science The presence of trained and equipped on-scene responders (e.g. athletic trainers, coaches) would enable effective initial care during the interval until EMS arrival, and improve patient outcomes.

\section{CAN EXPOSURE TO FRAMED MESSAGES ABOUT SAFETY REDUCE RISK BEHAVIOURS BY SCHOOL-AGED CHILDREN?}

Mackenzie Seasons, Emily Weinberger, Barbara Morrongiello. University of Guelph, Ontario Canada

\subsection{6/injuryprev-2020-savir.119}

Statement of Purpose The current study aimed to determine whether delivering framed safety messages (gain-framed, lossframed, and no frame) in poster format reduced physical risktaking behaviours when children were in a positive mood.
Previous research has shown that when in an elevated positive mood state, children engage in more risk behaviours than when in a neutral mood state, which leads to greater risk of injury (Morrongiello et al., 2014). Research in this area is particularly important for school-aged children, who are becoming increasingly independent and more vulnerable to injury outside of the home (Morrongiello et al., 2013).

Method/Approach 28 children (7-9 years old) were exposed to a message (gain-frame, loss-frame, or no frame (control) message) regarding play behaviours on a specific risk-taking measure (an obstacle course). Children's risk-taking was measured before and after a positive mood has been induced, and the impact of framed safety messaging on risk-taking behaviours was examined.

Results Results indicated the positive mood induction was successful and led to increased risk taking when participants were in a positive mood. Both gain- and loss- framed messages differentially counteracted this mood effect and led to reduced risk taking, but the loss-framed message reduced risky behaviours to a level significantly lower than the participants' baseline risk-taking behaviours.

Conclusions Results demonstrate that even in a positive mood, children can be influenced to engage in safer play behaviours with the use of message framing (particularly loss-framed messaging).

Significance and Contributions to Injury and Violence Prevention Science Given that physical risk taking was mitigated by framed safety posters (particularly loss-framed posters) even when children were in a positive mood, framed posters may be a cost-effective and useful intervention in places like public parks, where children are often happy and inclined to engage in increased risk-taking.

\section{7 A META-ANALYSIS ASSESSING THE OUTCOME OF OCCUPATIONAL INJURY BY MINORITY EMPLOYEE RACE}

Chelsea Hicks, Niclette Kibibi, William Thomson. University of lowa College of Public Health

10.1136/injuryprev-2020-savir.120

Statement of Purpose Literature suggests that racial disparities in the prevalence of occupational injury exist, but the magnitude of these disparities has not been summarized or welldescribed. The purpose of this meta-analysis was to examine differences of experienced workplace injury between White and Black employees.

Methods Our study population of interest was employees, teens to adults, who identified as non-Hispanic Black or nonHispanic white, and who experienced an unintentional injury in the workplace. PubMed and Embase were searched, which resulted in a total of 488 published articles. Articles were identified and reviewed using search terms related to workplace injuries and racial ethnic disparities. Standard meta-analysis tools were used to rate the quality of the studies and data combined using generic inverse variance pooling. Random effects models were used to account for the heterogeneity between the studies.

Results A total of 5 articles met eligibility criteria and included workplace injury information from both national databases and smaller assessments. Publication dates ranged from 2003 to 2017 with cross sectional and cohort studies present. When assessing the outcome of injury for those who are a racial/ethnic minority, individuals who were African American were 